\title{
KRÄUTER, 7 ZWERGE, FREQUENZEN
}

\section{Sehr geehrte Frau Kollegin, Sehr geehrter Herr Kollege,}

wir Österreicher bemühen uns, kreativ zu sein und das therapeutische Repertoire ständig zu erweitern. Das aktuelle Heft soll dieses Bemühen widerspiegeln: Ich darf Ihnen nämlich wieder einmal ein „Österreicher-Heft“ vorstellen. Auf den folgenden Seiten finden Sie Therapieergänzungen, die immer dann zum Tragen kommen, wenn die normale Akupunktur nicht ausreichend wirkt. Gerade diese PatientInnen fordern unsere Fähigkeiten heraus und gerade sie zeigen uns auf, dass möglichst ganzheitliche Ansätze auch in therapierefraktären Fällen zum Ziel führen können. Der weite Blick auf unterschiedlichste Methoden ist ohnehin das Besondere dieser Zeitschrift, wenn man an Störherddiagnostik, Blütentherapie, Energiemeridian und Ähnliches denkt.

Wir wollen Ihre Aufmerksamkeit auf drei Säulen lenken, die bei uns in Österreich eine herausragende Rolle spielen:

Erstens die Weiterentwicklungen der Aurikulomedizin im Rahmen der „Frequenziellen Akupunktur“ von Dr. Manfred Reininger, der unermüdlich neueVorgangsweisen erarbeitet, die immer noch effizienter und praxisnäher sein sollen.

Zweitens die TCM-basierte Akupunktur, die diesmal von Dr. Olivia Krammer-Pojer mit Bezug zum Klimak- terium und seinen unterschiedlichen Erscheinungen mithilfe der 7 menopausalen Zwerge vorgestellt wird.

Drittens mein persönliches Steckenpferd: die Anwendung von westlichen Kräutern in Kombination mit Akupunktur und Aurikulomedizin, die von den PatientInnen sehr gerne aktiv angenommen wird und wesentlich zur Nachhaltigkeit der Therapie beitragen kann.

Wir hoffen, Sie mit unseren Beiträgen zu inspirieren, zum weiteren Nachlesen anzuregen und vor allem zum Erproben ermuntern zu können.

Zum Abschluss darf ich Ihnen im Namen meines Teams von der OGKA ganz herzlich eine ruhige, besinnliche Weihnachtszeit und einen guten Start in das Neue Jahr wünschen

Ihr

Prof. Dr. med. Leopold Dorfer

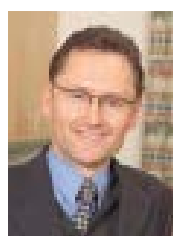

Prof. Dr. med. Leopold Dorfer

Präsident der Österreichischen Gesellschaft für Kontrollierte Akupunktur und TCM (OGKA)

Glacisstraße 7, A-8010 Graz

Tel. $+43316 / 374050$

E-Mail: office@ogka.at, Internet:www.ogka.at 